\title{
Using Metaheuristics in a Parallel Computing Course $^{\star}$
}

\author{
Ángel-Luis Calvo, Ana Cortés, Domingo Giménez ${ }^{\star \star}$, and Carmela Pozuelo \\ Departamento de Informática y Sistemas, Universidad de Murcia, Spain \\ angelluiscalvo@gmail.com, acc8@alu.um.es, domingo@dif.um.es, \\ carmela@pozuelo.org
}

\begin{abstract}
In this paper the use of metaheuristics techniques in a parallel computing course is explained. In the practicals of the course different metaheuristics are used in the solution of a mapping problem in which processes are assigned to processors in a heterogeneous environment, with heterogeneity in computation and in the network. The parallelization of the metaheuristics is also considered.
\end{abstract}

\section{Introduction}

This paper presents a teaching experience in which metaheuristic and parallel computing studies are combined. A mapping problem is proposed to the students in the practicals of a course of "Algorithms and parallel programming" [1]. The problem consists of obtaining an optimum processes to processors mapping on a heterogeneous system. The simulated systems present heterogeneity both in the computational and network speeds, and the processes to map constitute a homogeneous set, which means a HoHe (Homogeneous processes in Heterogeneous system) model is represented [2]. The mapping problem is NP [3. Each student must propose the solution of the mapping problem with some metaheuristic.

The paper is organized in the following way: section 2 explains the course in which the experience has been carried out; section 3 presents the mapping problem; in section 4 the application of some of the metaheuristics is explained, including their parallelization; a test is given to the students to see how the teaching objectives have been fulfilled, and the results of the test are commented in section 5; finally, section 6 summarizes the conclusions and outlines possible future studies.

\section{Organization of the Course}

The course is part of the fifth year of the studies in Computer Science, at the University of Murcia, in Spain. The students had studied Algorithms and Data

\footnotetext{
* This work has been funded in part by the Consejería de Educación de la Comunidad de Murcia, Fundación Séneca, project number 02973/PI/05.

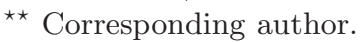


Structures, Computer Architecture, Concurrent Programming and Artificial Intelligence. The course is optional, so the students are high level students who are interested in the subject. This, together with the fact that a reduced number of students (approximately fifteen per year) take the course, means that the teaching is personalized and focused on the work of the students. They do different studies and practicals: preparation of a presentation about some algorithmic technique, both sequential and parallel; solution and theoretical and experimental study of an algorithm to solve a challenging problem sequentially; and obtaining parallel versions (in shared memory with OpenMP and in messagepassing with MPI) of the sequential algorithms. The course lasts one semester and it has sequential and parallel parts, which means the parallelism is studied in approximately two months. This reduced time together with the difficulty of an initial approach to parallelism means that the goal is to introduce the students to the problems and tools of parallelism, but we do not expect them to be able to develop new algorithms and carry out detailed experiments, but they must study and program available algorithms, to adjust them to the proposed problem, to design significant experiments and to draw valid conclusions. Thus, the topics of the course are: Introduction to complexity of problems, Tree traversal methods, Probabilistic algorithms, Metaheuristics, Matricial algorithms, Models of parallel programming, Analysis of parallel algorithms and Parallel algorithms.

First, the difficulties to solve some problems in a reduced time are stated. Then, some approximate, heuristics or numerical sequential algorithms are studied, and finally, the basics of parallel programming are analysed. Each student will develop sequential and parallel algorithms for the solution of a challenging problem. The proposed problem is a mapping problem where a set of identical processes is assigned to processors in a heterogeneous system. So, the students tackle a challenging problem in the field of parallel programming, and they work with topics in two parts (sequential approximate methods and parallel computing) of the syllabus. The methods proposed to solve this problem are: Backtracking or Branch and Bound with pruning based on heuristics (possibly pruning nodes which would lead to the optimum solution), Backtracking with tree traversal guided by heuristics, Probabilistic algorithms, Hill climbing, Tabu search, Scatter search, Genetic algorithms, Ant colony, Simulated annealing and GRASP. There are a lot of books on algorithms [56] and metaheuristics [7/8 which can be consulted by the students.

Each student makes two presentations: one on the general ideas of the technique assigned, and the other on the parallelization with OpenMP and MPI of some algorithm which implements this technique. The presentations are previous to the practical work, so that the students can exchange ideas about some parts of the problem (the representation of solutions and nodes, the general scheme of the algorithms, schemes of metaheuristics, possible combinations of techniques, ...) The collaboration of the students is fostered. The experimental comparison of the different techniques developed by the students is positively valued in the final evaluation of the practical. Additionally, at least two individual tutorials with each student would be organized, prior to each presentation. 


\section{The Assignation Problem}

The problem proposed is a simplified version of a mapping problem in which the execution time of a parallel homogeneous algorithm (all the processes work with the same amount of data and have the same computational cost) is used to obtain the mapping in a heterogeneous system with which the lowest possible execution time is achieved. The method was proposed in [9]. It is explained (simplified) to the students after the study of the topics about problem complexity, probabilistic algorithms and metaheuristics, and the papers in which the method was introduced and applied, along with other related papers, is made available to students. The method is summarized below.

The execution time of a parallel algorithm is modelled as a function of some algorithmic and system parameters [10]:

$$
t(s)=f(s, A P, S P)
$$

where $s$ represents the problem size. The system parameters $(S P)$ represent the characteristics of the system, and can be the cost of an arithmetic operation, the start-up $\left(t_{s}\right)$ and the word-sending $\left(t_{w}\right)$ time of communications. The algorithmic parameters $(A P)$ can be modified to obtain faster execution times. Some typical parameters in homogeneous systems are the number of processors to use from those available, or the number of rows and columns of processes.

The execution time model considered has the form:

$$
t(s, D)=t_{c} t_{c o m p}(s, D)+t_{s} t_{\text {start }}(s, D)+t_{w} t_{w o r d}(s, D)
$$

where $D$ represents the number of processes used in the solution of the problem, $t_{c}$ the cost of a basic arithmetic operations, $t_{c o m p}$ the number of basic arithmetic operations, $t_{\text {start }}$ the number of communications and $t_{\text {word }}$ the number of data communicated. In a homogeneous system the values of $t_{c}, t_{s}$ and $t_{w}$ are the same in the different processors. In a heterogeneous system it is also necessary to select the number of processes to use and the number of processes assigned to each processor. These numbers are stored in $d=\left(d_{1}, d_{2}, \ldots, d_{P}\right)$, with $P$ being the number of processors. The costs of a basic arithmetic operation in each one of the processors in the system are stored in an array $t_{c}$ with $P$ components, where $t_{c_{i}}$ is the cost in processor $i$. And the costs of $t_{s}$ and $t_{w}$ between each pair of processors are stored in two arrays $t_{s}$ and $t_{w}$ of sizes $P \times P$, and $t_{s_{i j}}$ and $t_{w_{i j}}$ are the start-up and word-sending times from processor $i$ to processor $j$.

The execution time model would be that of equation 2 , but with the values of $t_{c}, t_{s}$ and $t_{w}$ obtained from the formulae:

$$
t_{c}=\max \left\{d_{i} t_{c_{i}}\right\}, \quad t_{s_{i j}}=\max _{d_{i} \neq 0, d_{j} \neq 0}\left\{t_{s_{i j}}\right\}, \quad t_{w_{i j}}=\max _{d_{i} \neq 0, d_{j} \neq 0}\left\{t_{w_{i j}}\right\}
$$

In the model the cost of a basic operation in a processor is proportional to the number of processes in the processor, and no interferences are considered between processes in the same processor. 
Obtaining an optimum mapping becomes a tree traversal problem if we consider the tree of all the possible mappings. Figure 1 shows one such tree, with $P=3$. Each level represents the possible processors to which a process can be assigned. There is no limit to the height of the tree. Because the processes are all equal, the tree is combinatorial, and because more than one process can be assigned to a processor, it includes repetitions. The form of the logical tree, and the representation of the tree or the set to work with must be decided by the student. Each node in the solutions tree could be represented in at least two forms. In a representation with a value for each level, the grey node in figure 1 would be stated by $(1,2,2, \ldots)$. Because all the processes are equal, it is also possible to store the number of processes assigned to each processor. So, the grey node is represented by $(1,2,0)$.

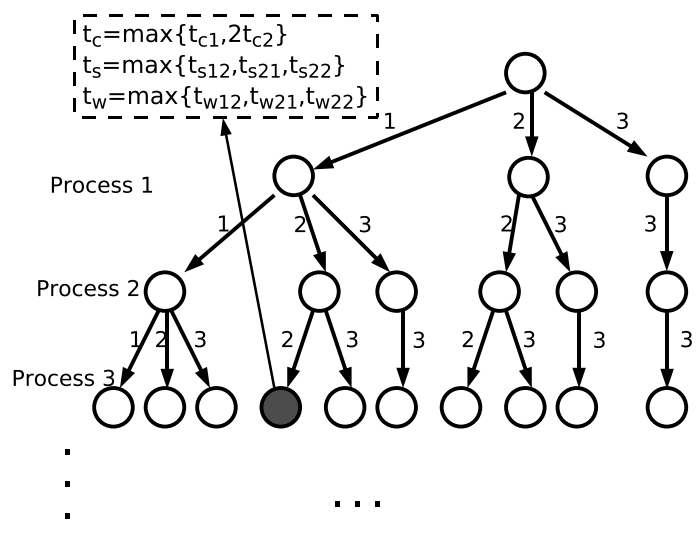

Fig. 1. Tree of the mappings of identical processes in a system with three processors

Sequential and parallel algorithms are developed and studied both theoretically and experimentally. The study would include the analysis of how the use of parallel computing contributes to reduce the execution time and/or the goodness of the solution. In order to have comparable results they must obtain experimental results with at least the functions:

$$
t_{c} \frac{n^{2}}{5 p}+t_{s} \frac{p(p-1)}{2}+t_{w} \frac{n(p-1)}{2}
$$

which corresponds to a parallel dynamic programming scheme [9], and:

$$
t_{c}\left(\frac{2}{3} \frac{n^{3}}{p}+\frac{n^{2}}{\sqrt{p}}\right)+t_{s} 2 n \sqrt{p}+t_{w} \frac{2 n^{2}}{\sqrt{p}}
$$

which corresponds to a parallel LU decomposition 11. The experiments should be carried out with the values in the ranges: $1<t_{c}<5,4<t_{w}<40$ and $20<t_{s}<100$. Small values of $t_{s}$ and $t_{w}$ would simulate the behaviour of shared memory multicomputers, medium values would correspond to distributed memory multicomputers, and large values to distributed systems. 


\section{Application of Metaheuristics to the Mapping Problem}

In this section the results obtained with three of the methods are shown. Two of the methods are metaheuristic methods (genetic algorithms and tabu search) and the other is a backtracking with pruning based on heuristics. In the sequential algorithms the stress is put on the high algorithmic representation which allows us to obtain different versions only by changing a routine in the scheme. For metaheuristic techniques a general scheme is studied [12. One such scheme is shown in algorithm 1 .

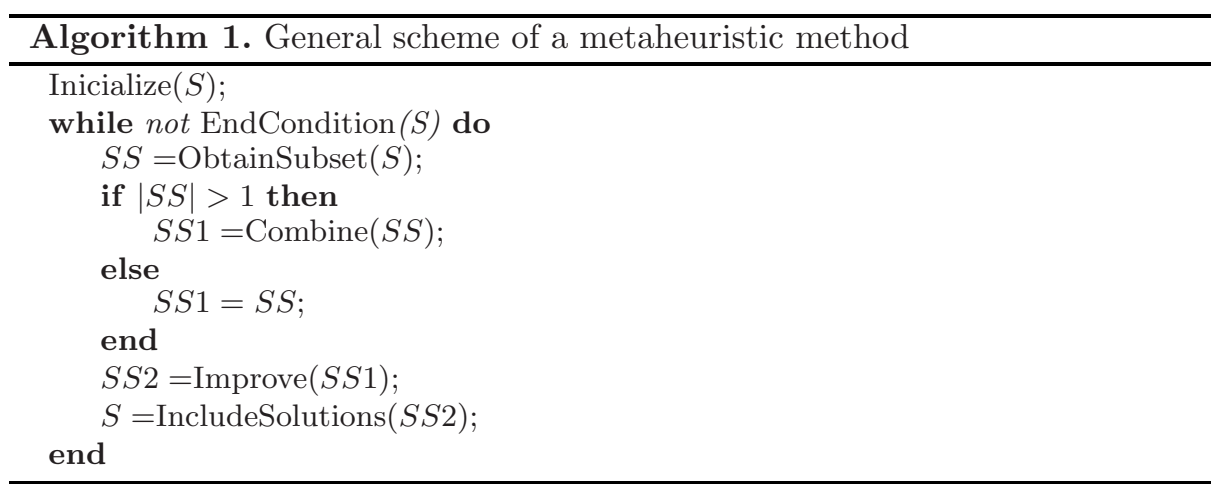

\subsection{Backtracking with Node Pruning}

Backtracking methods were used for this mapping problem in 9. For the simulation of small systems, backtracking was satisfactory, but for large systems huge assignation times were necessary. So, the work of the student was:

- For the sequential method:

- To understand the mapping problem and the increment of the assignation time when backtracking is used for large systems, which makes the backtracking impractical in most cases.

- To develop a backtracking scheme for the proposed mapping problem. The scheme should include a pruning routine which should be easy to substitute to experiment with different pruning techniques.

- To identify possible techniques to eliminate nodes which in some of the cases would not lead to the optimum mapping. The most representative techniques were:

PT1 The tree is searched until a maximum level, and nodes are not pruned. This method is included as a reference which ensures the optimum mapping.

PT2 At each step of the execution the lowest value $(G L V)$ of the modelled execution time of the nodes generated is stored. To decide if a node is pruned a "minimum value" $(N M V)$ is associated to it. 
When $N M V>G L V$ the node is pruned. In a node corresponding to $p$ processes, $N M V$ is obtained with a greedy method. From the execution time associated to the node, new values are obtained by substituting in the model $p$ by $p+1, p+2, \ldots$ while the value decreases. $N M V$ is taken as the minimum of these values.

PT3 $N M V$ is calculated in a node by substituting in the formula the value of the number of processes for the maximum speed-up achievable. For example, in node $(0,2,0)$, with a tree like that in figure 1] the first processor will not participate in the computation, and with $t_{c}=$ $(1,2,4)$, the relative speed-ups would be $s_{r}=(1,0.5,0.25)$, and the maximum achievable speed-up is 0.75 .

PT4 The same value as in the previous case is used for $p$ in the computation part, and the communication part does not vary.

- To carry out experiments to compare the results obtained with the different pruning techniques. Initially, experiments were carried out for small simulated systems (between 10 and 20 processors). The best results were obtained with PT3. This technique was used in successive experiments. The main conclusion was that for small systems backtracking with pruning can be used without a large execution time and obtaining a modelled time not far from the optimum. For big systems, the mapping time is too large to be applied in a real context. Parallelism could contribute to reduce the mapping time, so making the technique applicable.

- Different schemes were considered to obtain parallel versions, and finally a master-slave scheme was used:

- An OpenMP version is obtained in the following way: the master generates nodes until a certain level; slaves are generated and all the threads do backtracking from the nodes assigned cyclically to them.

- The MPI version works in the same way, but in this case the master processor sends nodes to the slave processors and these send back the results to the master.

- The sequential and parallel versions are compared. There is no important variation in the modelled time. The speed-up achieved is far from the optimum, and this is because independent backtrackings are carried out, which means less nodes are pruned with the parallel programs.

\subsection{Genetic Algorithm}

Genetic algorithms are possibly the most popular metaheuristic techniques. The students saw this technique in a previous course on Artificial Intelligence. The work of the student was:

- For the sequential method:

- To understand the mapping problem and to identify population and individual representations to apply genetic algorithms to the problem, to identify the possible forms of the routines in algorithm 1 for the genetic scheme, and to develop a genetic scheme at a high level. The scheme must allow easy changing of some parameters or routines. 
- To experimentally tune the values of the parameters and the routines to the mapping problem. The main conclusion was that to obtain a reduced assignation time it is necessary to reduce the number of individuals and the number of iterations, but on the other hand this reduction would produce a reduction in the goodness of the solution. Satisfactory results (both for the assignation time and the goodness of solution) are obtained from experiments with 10 individuals and with convergence after 10 iterations without improvement. In any case, genetic algorithms do not seem to be the most adequate metaheuristic for this problem.

- About the parallel versions:

- The OpenMP program works by simply parallelizing the combination of the population.

- From different parallel genetic schemes [13, the island scheme was selected for the message-passing version. The number of generations to exchange information between the islands is one parameter to be tuned.

- The sequential and parallel versions are compared. With OpenMP the same mappings are found, but with an important reduction in the assignation time. In MPI this time is not reduced substantially, but better mappings are normally obtained.

\subsection{Tabu Search}

Tabu search is a local search technique which uses memory structures to guide the search. The students saw this technique in a previous course on Artificial Intelligence. The work of the student was:

- For the sequential method:

- To understand the mapping problem and to identify set and element representations to apply tabu search to the problem, to identify how the routines in algorithm 1 would be for tabu search, and to develop a tabu search at a high level. The scheme must allow easy change of some parameters or routines.

- To experimentally tune the values of the parameters and the routines to the mapping problem. Satisfactory results were obtained when: the number of iterations a movement is tabu is equal to half the number of simulated processors $(P)$; the initial stage is obtained by assigning $P$ processes to the fastest processors; the number of iterations to begin the diversification phase is three quarters of the maximum number of iterations.

- About the parallel versions:

- The OpenMP program works by selecting a number of nodes to explore at each step equal to the number of available processors.

- For the MPI version, different tabu techniques have been studied 14 . A pC/RS/MPDS technique was used: each process controls its own search; knowledge is not shared by the processes; multiple initial solutions; and different search strategies are used. To diversify the search, 
some processes start with heuristic solutions and others with random solutions, and the number of iterations a movement is tabu depends on the number of the process.

- The sequential and parallel versions are compared. In OpenMP the speedup is satisfactory. In MPI the time is not reduced substantially, and only a small improvement in the mappings is obtained. A small reduction in the execution time can be achieved by reducing the number of iterations.

\section{$5 \quad$ Evaluating Teaching}

In order to evaluate if the teaching objectives have been fulfilled a test has been prepared. The test has seven statements. In some of them a high value is positive, but in others the answer is more positive when the value is lower. In that way, the test must be completed by reading it carefully. Each item is valued from 1 to 5, with 1 meaning total disagreement and 5 total agreement. Two questions are analyzed in the test: is the combination of the study of sequential approximation methods and of parallel programming appropriate? is it interesting to use a problem to guide the teaching?

The statements in the test are:

1. It is suitable to combine the study of sequential algorithms with the study of parallel computing because in that way two methods to solve high cost problems are studied together.

2. The combination in a course of the study of sequential methods with parallel programming means the course is overloaded, and it would be preferable to have two different courses to study the two subjects.

3. The use of a mapping problem in parallel computing and tackling the problem with heuristic methods is useful to clarify ideas about some methods previously studied in other courses.

4. The mapping problem has made the study of parallel programming difficult, because the problem deals with heterogeneous systems and the parallel programming practicals have been done in homogeneous systems.

5. The proposal of the mapping problem at the beginning of the course has been useful because it has motivated and guided the study of parallel programming.

6. The combination of the study of sequential methods and parallel programming has made it more difficult to follow the course because it has not been possible to study a theme in depth before beginning the next one.

7. The use of a problem to guide the teaching is appropriate because it motivates the study of each one of the themes.

Table 1 shows the positive or negative rating of each item in the test in relation to the two questions.

The number of students in the course is low. So, no significant conclusions can be drawn from the answers, but some indicators can be observed. Table 2 
Table 1. Positive or negative rating of each item in relation with the two questions to be evaluated

\begin{tabular}{|c|c|c|c|c|c|c|c|}
\hline item & 1 & 2 & 3 & 4 & 5 & 6 & 7 \\
\hline Heuristic-Parallel & + & - & + & - & & - & \\
\hline Problem-Guided & & & + & - & + & & + \\
\hline
\end{tabular}

summarizes the answers. The mean value for each item is shown. Also the means of the questions about the join study of approximation methods and parallel programming (H-P) and about the use of a problem to guide the course ( $\mathrm{P}$ $\mathrm{G})$ are shown. To obtain the mean, in the negative items negative and positive values are interchanged ( 1 for 5 and 2 for 4 ). The conclusion is that the two teaching objectives have been successfully fulfilled.

Table 2. Mean answer for each item and question in the test

\begin{tabular}{|c|c|c|c|c|c|c|c|c|}
\hline 1 & 2 & 3 & 4 & 5 & 6 & 7 & H-P & P-G \\
\hline 3.57 & 3.14 & 4.43 & 2.71 & 3.57 & 3.14 & 4.00 & 3.40 & 3.82 \\
\hline
\end{tabular}

\section{Conclusions and Possible Future Studies}

The paper presents a teaching experience using metaheuristics in combination with parallel computing in a course of "Algorithms and Parallel Programming". With this combination the students work at the same time with two of the topics of the course, the importance of approximate methods and heuristics is better understood when working with a challenging problem like the one proposed, and the difficulty and importance of the mapping problem is better understood when working on the problem with a metaheuristic approach. Furthermore, parallel programming is introduced using the same metaheuristics with which the mapping problem is tackled, and parallelism at different levels and in shared memory and message-passing is considered. In addition, because all the students work with the same mapping problem, but each student works with a different mapping algorithm, collaboration between students and common enrichment is fostered.

The success of the course organization has been evaluated through a test for the students. The preliminary experience seems to be very positive, and so it will be continued in successive courses. At the moment other mapping problems in the field of parallel computing are being considered.

\section{References}

1. Giménez, D.: Web page of the Algorithms and Parallel Programming course at the University of Murcia, http://dis.um.es/ domingo/app.html

2. Kalinov, A., Lastovetsky, A.: Heterogeneous distribution of computations while solving linear algebra problems on network of heterogeneous computers. Journal of Parallel and Distributed Computing 61(44), 520-535 (2001) 
3. Lennerstad, H., Lundberg, L.: Optimal scheduling results for parallel computing. In: SIAM News, pp. 16-18. SIAM, Philadelphia (1994)

4. Brucher, P.: Scheduling Algorithms, 5th edn. Springer, Heidelberg (2007)

5. Brassard, G., Bratley, P.: Fundamentals of Algorithms. Prentice-Hall, Englewood Cliffs (1996)

6. Cormen, T.H., Leiserson, C.E., Rivest, R.L.: Introduction to Algorithms. MIT Press, Cambridge (1990)

7. Dréo, J., Pétrowski, A., Siarry, P., Taillard, E.: Metaheuristics for Hard Optimization. Springer, Heidelberg (2005)

8. Hromkovič, J.: Algorithmics for Hard Problems, 2nd edn. Springer, Heidelberg (2003)

9. Cuenca, J., Giménez, D., Martínez-Gallar, J.P.: Heuristics for work distribution of a homogeneous parallel dynamic programming scheme on heterogeneous systems. Parallel Computing 31, 717-735 (2005)

10. Cuenca, J., Giménez, D., González, J.: Architecture of an automatic tuned linear algebra library. Parallel Computing 30(2), 187-220 (2004)

11. Cuenca, J., García, L.P., Giménez, D., Dongarra, J.: Processes distribution of homogeneous parallel linear algebra routines on heterogeneous clusters. In: Proc. IEEE Int. Conf. on Cluster Computing, IEEE Computer Society Press, Los Alamitos (2005)

12. Raidl, G.R.: A unified view on hybrid metaheuristics. In: Almeida, F., Blesa Aguilera, M.J., Blum, C., Moreno Vega, J.M., Pérez Pérez, M., Roli, A., Sampels, M. (eds.) HM 2006. LNCS, vol. 4030, pp. 1-12. Springer, Heidelberg (2006)

13. Luque, G., Alba, E., Dorronsoro, B.: Parallel genetic algorithms. In: Alba, E. (ed.) Parallel Metaheuristics (2005)

14. Crainic, T.G., Gendreau, M., Potvin, J.Y.: Parallel tabu search. In: Alba, E. (ed.) Parallel Metaheuristics (2005) 